\title{
Estimation of Hourly Solar Radiation on Horizontal and Inclined Surfaces in Western Himalayas
}

\author{
Shyam S. Chandel ${ }^{1}$, Rajeev K. Aggarwal $^{2}$ \\ ${ }^{1}$ Centre of Excellence for Energy \& Environment, NIT, Hamirpur, India; ${ }^{2}$ Department of Environmental Science, University of Hor- \\ ticulture \& Forestry, Nauni, India. \\ Email: chandel_shyam@yahoo.com, rajeev1792@rediffmail.om
}

Received November 25 ${ }^{\text {th }}$, 2010; revised January $12^{\text {th }}$, 2011, accepted January $16^{\text {th }}, 2011$.

\begin{abstract}
The hourly solar radiation data required for solar energy system design evaluation and performance studies is generally not available for a number of sites especially in remote locations. As such accurate determination of hourly solar radiation data, is important both at horizontal; surfaces and inclined surfaces. A model to estimate global solar radiation using temperature and sunshine hour data has been developed (Chandel et al. [1]) which is used to calculate the hourly solar radiation Data. The hourly solar radiation has also been calculated using Gueymard [2] daily integration approach from the measured daily solar radiation data. These two predicted hourly solar radiation data values are compared with measured hourly values to test the accuracy of the models. The total solar radiation on the inclined surfaces and vertical surfaces for different orientations, have also been estimated. The estimated values are found to be in close agreement with measured values. The method presented can be used to estimate hourly, global, diffuse solar radiation for horizontal surfaces and total solar radiation on inclined and vertical surfaces at different orientations with greater accuracy for any location.
\end{abstract}

Keywords: Solar Energy, Hourly Solar Radiation, Diffuse Solar Radiation

\section{Introduction}

The measured hourly values of solar radiation data are not available for large number of stations. Thus, accurate estimation of hourly values of global and diffused solar radiation data is essential for the design and performance evaluation of solar energy systems. The hourly solar radiation can be estimated using Gueymard [2] daily integration approach. It requires measured value of daily solar radiation, which is not available for most of the locations. This can be estimated by using sunshine hour data or temperature data. A model has been developed based on latitude and altitude of a location to estimate monthly average global solar radiation using sunshine hour and temperature data (Chandel et al. [1]). The values of solar radiation obtained using this model are used to estimate hourly solar radiation following Gueymard [2] daily integration approach. In order to verify the accuracy of the results obtained, the hourly solar radiation is also estimated from the measured daily solar radiation data for Delhi.
This method is first used to predict hourly global solar radiation for Delhi [Latitude $28.58^{\circ} \mathrm{N}$, Longitude $77.2^{\circ} \mathrm{E}$, altitude $216 \mathrm{~m}$ (amsl)] for which 10-15 years measured data are available. Establishing the accuracy, the model is then used to calculate the values for location like Shimla [Latitude $\left(31.1^{\circ} \mathrm{N}\right)$, Longitude $\left(77.1^{\circ} \mathrm{E}\right)$ and altitude of $2002 \mathrm{~m}$ (amsl)] for which the measured data are not available.

The hourly diffuse solar radiation for Delhi has also been calculated using correlation given by Orgill and Hollands [3] with modified value of regression coefficient ' $c$ ' in Section III. The total hourly value of solar radiation on inclined south surfaces and vertical surfaces at different orientations has been estimated for Delhi in Section IV. The relation given by Klein [4] does not predict accurate results on north orientation, which is modified particularly for the months with negative declination.

\section{Hourly Global Solar Radiation}

The daily average extra terrestrial irradiation on a hori- 
zontal surface Go is given as

where $k=24 / \pi$,

$$
G_{o}=k \omega_{s} R G_{s c} \sinh _{o}
$$

$$
\cos \omega_{s}=-\tan \phi \tan \delta
$$

$\phi$ is negative for Southern hemisphere, $h_{o}$ is given as

$$
\sin h_{o}=q A\left(\omega_{s}\right) / \omega_{s}
$$

with

$$
q=\cos \phi \cos \delta
$$

and

$$
A\left(\omega_{s}\right)=\sin \omega_{s}-\omega_{s} \cos \omega_{s}
$$

where

$$
\text { So }=k \omega_{s}
$$

$$
r_{t}=r_{o}\left[1+q\left(a_{2} / a_{1}\right) k A\left(\omega_{s}\right) r_{o}\right] /\left[1+q\left(a_{2} / a_{1}\right) B\left(\omega_{s}\right) / A\left(\omega_{s}\right)\right]
$$

where

$$
\begin{gathered}
a_{1}=0.41341 K_{t}+0.61197 K_{t}^{2}-0.01886 K_{t} S_{o}+0.00759 S_{o} \\
a_{2}=\operatorname{Max}\left(0.054,0.28116+2.2475 K_{t}-1.76118 K_{t}^{2}-1.84535 \sin h_{o}+1.6811 \sin ^{3} h_{o}\right)
\end{gathered}
$$

are dependent on both $K_{t}$ and $\omega_{s}$. Here $K_{t}$ is the ratio of irradiation measured at the earth surface to that calculated at the top of the atmosphere. In present calculation $a_{2}$ is calculated using 0.28116 in Equation (13), which gives maximum value of $a_{2}$ in comparison to 0.054 . In present study the values of $\mathrm{K}_{\mathrm{t}}$ has been used suggested by Gueymard [2] i.e. 0.610.

The mean hourly global solar radiation for Delhi [Table 1] has been calculated using Equations (9) and (11) with measured values of daily solar radiation, which reveals that the estimated values are close to the measured values for the period 1967-1978 [Mani [7]]. The mean hourly global solar radiation for Delhi [Table 2] has also been calculated using Equations (9) and (11) with estimated values of daily solar radiation using Chandel et al. [1] model, which reveals that the estimated values are close to the measured values except for the month of July and August. The percentage variation in the estimation of hourly values of solar radiation using measured daily values and predicted values by Chandel et al. [1] with measured values of hourly values of solar radiation has been presented in Table 3. This table reveals that predicted values by can be used for the estimation of hourly values of solar radiation except for the months of July and August where the percentage error is high. The model is used to calculate the hourly values for Shimla, which are presented in Table 4.

\section{Hourly Diffuse Solar Radiation}

The estimation of hourly diffuse solar radiation is essen- tial to calculate the direct radiation from the total solar radiation. A number of methods have been used to determine the hourly diffuse solar radiation. Liu and Jordan [6] developed a method to calculate mean hourly diffuse radiation from mean daily totals, which gives results with reasonably accuracy. Orgill and Hollands [3] have used the data from Canadian stations to correlate the diffuse hourly Solar radiation $D_{h}$ and total solar radiation $G_{h}$ with the hourly clearness index, $k_{T}$ [ratio of the hourly global radiation to the hourly extra terrestrial radiation]. Following this approach, the equation for the correlation is written as

$$
D_{h} / G_{h}=\left(c+d k_{T}\right)
$$

The hourly $G_{o h}$ on a horizontal surface has been calculated using the relation Duffie \& Beckman [8]

$$
\begin{aligned}
G_{o h} & =G_{s c} 24 / \pi k\left(\left(\omega_{2}-\omega_{1}\right) \sin \phi \sin \delta\right. \\
& \left.+\left(\sin \omega_{2}-\sin \omega_{1}\right) \cos \phi \cos \delta\right)
\end{aligned}
$$

$\omega_{2}$ and $\omega_{1}$ are the hour angle where $\omega_{2}>\omega_{1}$.

$$
k_{T}=G_{h} / G_{o h}
$$

The value of regression coefficient $d$ is chosen as -0.249 , whereas the value of $c$ is taken for different months using Delhi data as:

Jan. Feb. Mar. Apr. May Jun. Jul. Aug. Sep. Oct. Nov. Dec.

$\begin{array}{llllllllllll}0.37 & 0.36 & 0.36 & 0.41 & 0.42 & 0.55 & 0.61 & 0.60 & 0.42 & 0.33 & 0.32 & 0.35\end{array}$ 
Table 1. Measured* and estimated Hourly global solar radiation $\left(\mathrm{kwh} / \mathrm{m}^{2}\right)$ using measured daily values for Delhi.

\begin{tabular}{|c|c|c|c|c|c|c|c|c|c|c|c|c|}
\hline LAT & Jan. & Feb. & Mar. & Apr. & May & Jun. & Jul. & Aug. & Sep. & Oct. & Nov. & Dec. \\
\hline 5.30 & & & & & & & & & & & & $\begin{array}{c}0.000 \\
(0.000)\end{array}$ \\
\hline 6.30 & $\begin{array}{c}0.000 \\
(0.004)\end{array}$ & $\begin{array}{c}0.002 \\
(0.020)\end{array}$ & & & & & & & & & & $\begin{array}{c}0.000 \\
(0.003)\end{array}$ \\
\hline 7.30 & $\begin{array}{c}0.113 \\
(0.096)\end{array}$ & $\begin{array}{c}0.177 \\
(0.163)\end{array}$ & $\begin{array}{c}0.264 \\
(0.260)\end{array}$ & $\begin{array}{c}0.335 \\
(0.339)\end{array}$ & $\begin{array}{c}0.389 \\
(0.383)\end{array}$ & & $\begin{array}{c}0.271 \\
(0.275)\end{array}$ & & & & & $\begin{array}{c}0.093 \\
(0.085)\end{array}$ \\
\hline 8.30 & $\begin{array}{c}0.292 \\
(0.271)\end{array}$ & $\begin{array}{c}0.367 \\
(0.364)\end{array}$ & $\begin{array}{c}0.466 \\
(0.475)\end{array}$ & $\begin{array}{c}0.535 \\
(0.554)\end{array}$ & $\begin{array}{c}0.583 \\
(0.581)\end{array}$ & & & & $\begin{array}{c}0.428 \\
(0.431)\end{array}$ & & & $\begin{array}{c}0.262 \\
(0.259)\end{array}$ \\
\hline 9.30 & $\begin{array}{c}0.466 \\
(0.433)\end{array}$ & $\begin{array}{c}0.545 \\
(0.540)\end{array}$ & $\begin{array}{c}0.652 \\
(0.655)\end{array}$ & $\begin{array}{c}0.718 \\
(0.727)\end{array}$ & $\begin{array}{c}0.759 \\
(0.746)\end{array}$ & $\begin{array}{c}0.665 \\
(0.657)\end{array}$ & & & $\begin{array}{c}0.594 \\
(0.587)\end{array}$ & & & \\
\hline 10.30 & $\begin{array}{c}0.604 \\
(0.556)\end{array}$ & $\begin{array}{c}0.684 \\
(0.670)\end{array}$ & $\begin{array}{c}0.795 \\
(0.781)\end{array}$ & $\begin{array}{c}0.858 \\
(0.848)\end{array}$ & & & & & & & & \\
\hline 11.30 & $\begin{array}{c}0.680 \\
(0.618)\end{array}$ & $\begin{array}{c}0.759 \\
(0.727)\end{array}$ & $\begin{array}{c}0.872 \\
(0.845)\end{array}$ & $\begin{array}{c}0.934 \\
(0.910)\end{array}$ & & $\begin{array}{c}0.845 \\
(0.809)\end{array}$ & & & $\begin{array}{c}0.792 \\
(0.757)\end{array}$ & & & \\
\hline 12.30 & $\begin{array}{c}0.680 \\
(0.615)\end{array}$ & $\begin{array}{c}0.759 \\
(0.728)\end{array}$ & $\begin{array}{c}0.872 \\
(0.844)\end{array}$ & $\begin{array}{c}0.934 \\
(0.903)\end{array}$ & $\begin{array}{c}0.966 \\
(0.920)\end{array}$ & $\begin{array}{c}0.845 \\
(0.813)\end{array}$ & $\begin{array}{c}0.686 \\
(0.671)\end{array}$ & $\begin{array}{c}0.658 \\
(0.659)\end{array}$ & $\begin{array}{c}0.792 \\
(0.747)\end{array}$ & & $\begin{array}{c}0.733 \\
(0.680)\end{array}$ & $\begin{array}{c}0.627 \\
(0.605)\end{array}$ \\
\hline 13.30 & $\begin{array}{c}0.604 \\
(0.552)\end{array}$ & $\begin{array}{c}0.684 \\
(0.659)\end{array}$ & $\begin{array}{c}0.795 \\
(0.769)\end{array}$ & $\begin{array}{c}0.858 \\
(0.833)\end{array}$ & $\begin{array}{c}0.893 \\
(0.844)\end{array}$ & $\begin{array}{c}0.782 \\
(0.750)\end{array}$ & $\begin{array}{c}0.633 \\
(0.606)\end{array}$ & & $\begin{array}{c}0.722 \\
(0.689)\end{array}$ & & & $\begin{array}{c}0.556 \\
(0.538)\end{array}$ \\
\hline 14.30 & $\begin{array}{c}0.466 \\
(0.430)\end{array}$ & $\begin{array}{c}0.545 \\
(0.534)\end{array}$ & & $\begin{array}{c}0.718 \\
(0.699)\end{array}$ & $\begin{array}{c}0.759 \\
(0.719)\end{array}$ & $\begin{array}{c}0.665 \\
(0.639)\end{array}$ & & & $\begin{array}{c}0.594 \\
(0.571)\end{array}$ & & & $\begin{array}{c}0.426 \\
(0.418)\end{array}$ \\
\hline 15.30 & $\begin{array}{c}0.292 \\
(0.268)\end{array}$ & $\begin{array}{c}0.367 \\
(0.358)\end{array}$ & $\begin{array}{c}0.466 \\
(0.458)\end{array}$ & $\begin{array}{c}0.535 \\
(0.523)\end{array}$ & $\begin{array}{c}0.583 \\
(0.547)\end{array}$ & $\begin{array}{c}0.511 \\
(0.483)\end{array}$ & $\begin{array}{c}0.409 \\
(0.412)\end{array}$ & $\begin{array}{c}0.377 \\
(0.392)\end{array}$ & $\begin{array}{c}0.428 \\
(0.417)\end{array}$ & & & $\begin{array}{c}0.262 \\
(0.255)\end{array}$ \\
\hline 16.30 & $\begin{array}{c}0.113 \\
(0.099)\end{array}$ & $\begin{array}{c}0.177 \\
(0.165)\end{array}$ & $\begin{array}{c}0.264 \\
(0.249)\end{array}$ & $\begin{array}{c}0.335 \\
(0.318)\end{array}$ & $\begin{array}{c}0.389 \\
(0.352)\end{array}$ & $\begin{array}{c}0.344 \\
(0.318)\end{array}$ & $\begin{array}{c}0.271 \\
(0.273)\end{array}$ & $\begin{array}{c}0.238 \\
(0.255)\end{array}$ & $\begin{array}{c}0.249 \\
(0.244)\end{array}$ & $\begin{array}{c}0.201 \\
(0.191)\end{array}$ & $\begin{array}{c}0.139 \\
(0.125)\end{array}$ & $\begin{array}{c}0.093 \\
(0.086)\end{array}$ \\
\hline 17.30 & $\begin{array}{c}0.000 \\
(0.005)\end{array}$ & $\begin{array}{c}0.002 \\
(0.021)\end{array}$ & $\begin{array}{c}0.071 \\
(0.064)\end{array}$ & $\begin{array}{c}0.143 \\
(0.122)\end{array}$ & $\begin{array}{c}0.202 \\
(0.158)\end{array}$ & $\begin{array}{c}0.183 \\
(0.159)\end{array}$ & $\begin{array}{c}0.140 \\
(0.141)\end{array}$ & $\begin{array}{c}0.108 \\
(0.107)\end{array}$ & $\begin{array}{c}0.083 \\
(0.074)\end{array}$ & $\begin{array}{c}0.022 \\
(0.033)\end{array}$ & $\begin{array}{c}0.000 \\
(0.010)\end{array}$ & $\begin{array}{c}0.000 \\
(0.003)\end{array}$ \\
\hline Total & $\begin{array}{c}4.309 \\
(3.987)\end{array}$ & $\begin{array}{c}5.069 \\
(5.001)\end{array}$ & $\begin{array}{c}6.240 \\
(6.138)\end{array}$ & $\begin{array}{c}7.048 \\
(6.935)\end{array}$ & $\begin{array}{c}7.624 \\
(7.287)\end{array}$ & $\begin{array}{c}6.706 \\
(6.544)\end{array}$ & $\begin{array}{c}5.381 \\
(5.334)\end{array}$ & $\begin{array}{c}4.979 \\
(5.053)\end{array}$ & $\begin{array}{c}5.734 \\
(5.602)\end{array}$ & $\begin{array}{c}5.351 \\
(5.355)\end{array}$ & $\begin{array}{l}4.743 \\
4.523)\end{array}$ & $\begin{array}{c}3.929 \\
(3.843)\end{array}$ \\
\hline
\end{tabular}

('measured values are in brackets).

Table 2. Estimated Hourly global solar radiation $\left(\mathrm{kwh} / \mathrm{m}^{2}\right)$ using daily values predicted by Chandel et al. (2004) for Delhi.

\begin{tabular}{|c|c|c|c|c|c|c|c|c|c|c|c|c|}
\hline Hour angle & Jan. & Feb. & Mar. & Apr. & May & Jun. & Jul. & Aug. & Sep. & Oct. & Nov. & Dec. \\
\hline 97.5 & 0.000 & 0.000 & 0.000 & 0.000 & 0.027 & 0.047 & 0.036 & 0.000 & 0.000 & 0.000 & 0.000 & 0.000 \\
\hline 82.5 & 0.000 & 0.002 & 0.060 & 0.123 & 0.170 & 0.190 & 0.179 & 0.138 & 0.079 & 0.017 & 0.000 & 0.000 \\
\hline 67.50 & 0.097 & 0.149 & 0.222 & 0.292 & 0.338 & 0.355 & 0.345 & 0.306 & 0.241 & 0.167 & 0.107 & 0.081 \\
\hline 52.50 & 0.251 & 0.315 & 0.397 & 0.471 & 0.514 & 0.527 & 0.518 & 0.483 & 0.416 & 0.334 & 0.263 & 0.231 \\
\hline 37.50 & 0.402 & 0.472 & 0.561 & 0.637 & 0.675 & 0.684 & 0.677 & 0.646 & 0.579 & 0.492 & 0.415 & 0.378 \\
\hline 22.50 & 0.522 & 0.597 & 0.688 & 0.764 & 0.799 & 0.803 & 0.798 & 0.771 & 0.705 & 0.616 & 0.535 & 0.497 \\
\hline 7.5 & 0.589 & 0.665 & 0.757 & 0.833 & 0.866 & 0.868 & 0.864 & 0.839 & 0.774 & 0.684 & 0.602 & 0.563 \\
\hline-7.5 & 0.589 & 0.665 & 0.757 & 0.833 & 0.866 & 0.868 & 0.864 & 0.839 & 0.774 & 0.684 & 0.602 & 0.563 \\
\hline-22.5 & 0.522 & 0.597 & 0.688 & 0.764 & 0.799 & 0.803 & 0.798 & 0.771 & 0.705 & 0.616 & 0.535 & 0.497 \\
\hline-37.5 & 0.402 & 0.472 & 0.561 & 0.637 & 0.675 & 0.684 & 0.677 & 0.646 & 0.579 & 0.492 & 0.415 & 0.378 \\
\hline-52.5 & 0.251 & 0.315 & 0.397 & 0.471 & 0.514 & 0.527 & 0.518 & 0.483 & 0.416 & 0.334 & 0.263 & 0.231 \\
\hline-67.5 & 0.097 & 0.149 & 0.222 & 0.292 & 0.338 & 0.355 & 0.345 & 0.306 & 0.241 & 0.167 & 0.107 & 0.081 \\
\hline-82.5 & 0.000 & 0.002 & 0.060 & 0.123 & 0.170 & 0.190 & 0.179 & 0.138 & 0.079 & 0.017 & 0.000 & 0.000 \\
\hline-97.5 & 0.000 & 0.000 & 0.000 & 0.000 & 0.027 & 0.047 & 0.036 & 0.000 & 0.000 & 0.000 & 0.000 & 0.000 \\
\hline $\begin{array}{c}\text { Total } \\
\text { (Measured) }\end{array}$ & $\begin{array}{c}3.721 \\
(3.987)\end{array}$ & $\begin{array}{c}4.399 \\
(5.001)\end{array}$ & $\begin{array}{c}5.370 \\
(6.138)\end{array}$ & $\begin{array}{c}6.241 \\
(6.935)\end{array}$ & $\begin{array}{c}6.779 \\
(7.287)\end{array}$ & $\begin{array}{c}6.948 \\
(6.544)\end{array}$ & $\begin{array}{c}6.832 \\
(5.334)\end{array}$ & $\begin{array}{c}6.367 \\
(5.053)\end{array}$ & $\begin{array}{c}5.590 \\
(5.602)\end{array}$ & $\begin{array}{c}4.620 \\
(5.355)\end{array}$ & $\begin{array}{c}3.842 \\
(4.523)\end{array}$ & $\begin{array}{c}3.498 \\
(3.843)\end{array}$ \\
\hline
\end{tabular}


Table 3. Percentage variation in estimation of hourly values using measured daily values* and using predicted daily solar radiation by Chandel et al.**.

\begin{tabular}{ccccccccccccc}
\hline & Jan. & Feb. & Mar. & Apr. & May & Jun. & Jul. & Aug. & Sep. & Oct. & Nov. & Dec. \\
\hline $\begin{array}{c}\text { Measured } \\
\left.\mathbf{( k W h} / \mathbf{m}^{2}\right)\end{array}$ & 3.987 & $(5.001)$ & $(6.138)$ & $(6.935)$ & $(7.287)$ & $(6.544)$ & $(5.334)$ & $(5.053)$ & $(5.602)$ & $(5.355)$ & $(4.523)$ & $(3.843)$ \\
$* *(\%)$ & 4.64 & 10.10 & 10.59 & 8.07 & 5.01 & 8.40 & 30.80 & 28.70 & 1.98 & 11.82 & 13.20 & 6.97 \\
$*(\%)$ & 8.08 & 1.36 & 1.66 & 1.63 & 4.62 & 2.48 & 0.88 & 1.46 & 2.36 & 0.07 & 4.86 & 2.24 \\
\hline
\end{tabular}

Table 4. Estimated Hourly global radiation $\left(\mathrm{kwh} / \mathrm{m}^{2}\right)$ for Shimla.

\begin{tabular}{lllllllllllll}
\hline $\mathbf{5 . 3 0}$ & Jan. & Feb. & Mar. & Apr. & May & Jun. & Jul. & Aug. & Sep. & Oct. & Nov. & Dec. \\
\hline $\mathbf{6 . 3 0}$ & 0.000 & 0.000 & 0.000 & 0.000 & 0.028 & 0.043 & 0.023 & 0.003 & 0.000 & 0.000 & 0.000 & 0.000 \\
$\mathbf{7 . 3 0}$ & 0.000 & 0.000 & 0.038 & 0.099 & 0.141 & 0.147 & 0.085 & 0.066 & 0.052 & 0.011 & 0.000 & 0.000 \\
$\mathbf{8 . 3 0}$ & 0.061 & 0.096 & 0.144 & 0.233 & 0.275 & 0.270 & 0.152 & 0.134 & 0.157 & 0.134 & 0.071 & 0.039 \\
$\mathbf{9 . 3 0}$ & 0.176 & 0.210 & 0.260 & 0.375 & 0.416 & 0.397 & 0.216 & 0.201 & 0.270 & 0.272 & 0.184 & 0.124 \\
$\mathbf{1 0 . 3 0}$ & 0.292 & 0.322 & 0.370 & 0.508 & 0.545 & 0.513 & 0.272 & 0.259 & 0.375 & 0.406 & 0.293 & 0.209 \\
$\mathbf{1 1 . 3 0}$ & 0.386 & 0.410 & 0.455 & 0.610 & 0.644 & 0.602 & 0.313 & 0.303 & 0.456 & 0.511 & 0.380 & 0.276 \\
$\mathbf{1 2 . 3 0}$ & 0.439 & 0.459 & 0.501 & 0.666 & 0.698 & 0.651 & 0.335 & 0.326 & 0.501 & 0.569 & 0.428 & 0.314 \\
$\mathbf{1 3 . 3 0}$ & 0.439 & 0.459 & 0.501 & 0.666 & 0.698 & 0.651 & 0.335 & 0.326 & 0.501 & 0.569 & 0.428 & 0.314 \\
$\mathbf{1 4 . 3 0}$ & 0.386 & 0.410 & 0.455 & 0.610 & 0.644 & 0.602 & 0.313 & 0.303 & 0.456 & 0.511 & 0.380 & 0.276 \\
$\mathbf{1 5 . 3 0}$ & 0.292 & 0.322 & 0.370 & 0.508 & 0.545 & 0.513 & 0.272 & 0.259 & 0.375 & 0.406 & 0.293 & 0.209 \\
$\mathbf{1 6 . 3 0}$ & 0.176 & 0.210 & 0.260 & 0.375 & 0.416 & 0.397 & 0.216 & 0.201 & 0.270 & 0.272 & 0.184 & 0.124 \\
$\mathbf{1 7 . 3 0}$ & 0.061 & 0.096 & 0.144 & 0.233 & 0.275 & 0.270 & 0.152 & 0.134 & 0.157 & 0.134 & 0.071 & 0.039 \\
$\mathbf{1 8 . 3 0}$ & 0.000 & 0.000 & 0.038 & 0.099 & 0.141 & 0.147 & 0.085 & 0.066 & 0.052 & 0.011 & 0.000 & 0.000 \\
$\mathbf{T o t a l}$ & $\mathbf{2 . 7 1 0}$ & $\mathbf{2 . 9 9 5}$ & $\mathbf{3 . 5 3 6}$ & $\mathbf{4 . 9 8 4}$ & $\mathbf{5 . 4 6 8}$ & $\mathbf{5 . 2 0 4}$ & $\mathbf{2 . 7 7 0}$ & $\mathbf{2 . 5 8 2}$ & $\mathbf{3 . 6 2 3}$ & $\mathbf{3 . 8 0 3}$ & $\mathbf{2 . 7 1 3}$ & $\mathbf{1 . 9 2 5}$ \\
\hline & & & & & & & & & &
\end{tabular}

The hourly diffuse solar radiation values for Delhi are given in Table 5 which reveals that the estimated values are close to the measured values Mani [7]. This method has been used to estimate the hourly diffuse values for Shimla (Table 6).

\section{Total Hourly Radiation on Inclined Surfaces}

The values of hourly total solar radiation at tilted surfaces are required for assessing the thermal performance of solar collectors; in solar passive heating and cooling of buildings as these systems/collectors are to be installed at inclined angles for maximum efficiency. Generally, radiation stations measure the solar radiation data on a horizontal surface so it is important to estimate the solar radiation at inclined surfaces for which global radiation tilt factor has to be calculated.

Klein [4] developed a method to calculate tilt factors using an isotropic model for computing diffuse sky radiation, following Liu \& Jordan [6], which was applicable only for true south. Robinson [9] and Revfeim [10] proposed models, which assume a part of sky radiation to be direct radiation and the remaining part as isotropic. In the present case we have used the model of Klein [4] to calculate tilt factor for surfaces of various surface azimuth angles. The hourly total radiation on tilted surface $G_{T h}$ is given as

$$
G_{T h}=G_{h} r
$$

where $r$ is global radiation tilt factor and is given by

$$
r=\left(1-D_{h} / G_{h}\right) r_{b}+r_{d}\left(D_{h} / G_{h}\right)+r_{r} \rho
$$

and

$$
r_{b}=\cos \theta / \cos \theta z
$$

The angle of incidence of beam radiation $\theta$, is related to other angles by relation:

$$
\begin{aligned}
\cos \theta= & \sin \phi(\sin \delta \cos \beta+\cos \delta \cos \gamma \cos \omega \sin \beta) \\
& +\cos \phi(\cos \delta \cos \omega \cos \beta-\sin \delta \cos \gamma \sin \beta) \\
& +\cos \delta \sin \gamma \sin \beta \sin \omega
\end{aligned}
$$


Table 5. Measured* and estimated Hourly diffuse solar radiation $\left(\mathrm{kwh} / \mathrm{m}^{2}\right)$ for Delhi.

\begin{tabular}{|c|c|c|c|c|c|c|c|c|c|c|c|c|}
\hline LAT & Jan. & Feb. & Mar. & Apr. & May & Jun. & Jul. & Aug. & Sep. & Oct. & Nov. & Dec. \\
\hline 5.30 & $\begin{array}{c}0.000 \\
(0.000)\end{array}$ & $\begin{array}{c}0.000 \\
(0.000)\end{array}$ & $\begin{array}{c}0.000 \\
(0.001)\end{array}$ & $\begin{array}{c}0.000 \\
(0.009)\end{array}$ & $\begin{array}{c}0.016 \\
(0.027)\end{array}$ & $\begin{array}{c}0.025 \\
(0.033)\end{array}$ & $\begin{array}{c}0.018 \\
(0.023)\end{array}$ & $\begin{array}{c}0.000 \\
(0.009)\end{array}$ & $\begin{array}{c}0.000 \\
(0.002)\end{array}$ & $\begin{array}{c}0.000 \\
(0.000)\end{array}$ & $\begin{array}{c}0.000 \\
(0.000)\end{array}$ & $\begin{array}{c}0.000 \\
(0.000)\end{array}$ \\
\hline 6.30 & $\begin{array}{c}0.000 \\
(0.003)\end{array}$ & $\begin{array}{c}0.001 \\
(0.013)\end{array}$ & $\begin{array}{c}0.025 \\
(0.043)\end{array}$ & $\begin{array}{c}0.056 \\
(0.083)\end{array}$ & $\begin{array}{c}0.081 \\
(0.114)\end{array}$ & $\begin{array}{c}0.097 \\
(0.124)\end{array}$ & $\begin{array}{c}0.083 \\
(0.096)\end{array}$ & $\begin{array}{c}0.063 \\
(0.074)\end{array}$ & $\begin{array}{c}0.034 \\
(0.053)\end{array}$ & $\begin{array}{c}0.007 \\
(0.026)\end{array}$ & $\begin{array}{c}0.000 \\
(0.007)\end{array}$ & $\begin{array}{c}0.000 \\
(0.002)\end{array}$ \\
\hline 7.30 & $\begin{array}{c}0.040 \\
(0.051)\end{array}$ & $\begin{array}{c}0.060 \\
(0.076)\end{array}$ & $\begin{array}{c}0.087 \\
(0.113)\end{array}$ & $\begin{array}{c}0.126 \\
(0.153)\end{array}$ & $\begin{array}{c}0.148 \\
(0.190)\end{array}$ & $\begin{array}{c}0.178 \\
(0.215)\end{array}$ & $\begin{array}{c}0.158 \\
(0.178)\end{array}$ & $\begin{array}{c}0.137 \\
(0.153)\end{array}$ & $\begin{array}{c}0.098 \\
(0.123)\end{array}$ & $\begin{array}{c}0.061 \\
(0.087)\end{array}$ & $\begin{array}{c}0.042 \\
(0.057)\end{array}$ & $\begin{array}{c}0.031 \\
(0.045)\end{array}$ \\
\hline 8.30 & $\begin{array}{c}0.095 \\
(0.103)\end{array}$ & $\begin{array}{c}0.114 \\
(0.126)\end{array}$ & $\begin{array}{c}0.143 \\
(0.159)\end{array}$ & $\begin{array}{c}0.190 \\
(0.202)\end{array}$ & $\begin{array}{c}0.211 \\
(0.244)\end{array}$ & $\begin{array}{c}0.256 \\
(0.286)\end{array}$ & $\begin{array}{c}0.233 \\
(0.257)\end{array}$ & $\begin{array}{c}0.211 \\
(0.224)\end{array}$ & $\begin{array}{c}0.159 \\
(0.178)\end{array}$ & $\begin{array}{c}0.110 \\
(0.128)\end{array}$ & $\begin{array}{c}0.090 \\
(0.099)\end{array}$ & $\begin{array}{c}0.081 \\
(0.092)\end{array}$ \\
\hline 9.30 & $\begin{array}{c}0.138 \\
(0.140)\end{array}$ & $\begin{array}{c}0.156 \\
(0.159(\end{array}$ & $\begin{array}{c}0.185 \\
(0.189)\end{array}$ & $\begin{array}{c}0.241 \\
(0.236)\end{array}$ & $\begin{array}{c}0.261 \\
(0.279)\end{array}$ & $\begin{array}{c}0.322 \\
(0.342)\end{array}$ & $\begin{array}{c}0.299 \\
(0.316)\end{array}$ & $\begin{array}{c}0.277 \\
(0.277)\end{array}$ & $\begin{array}{l}0.210 \\
(0.22)\end{array}$ & $\begin{array}{c}0.147 \\
(0.154)\end{array}$ & $\begin{array}{c}0.125 \\
(0.122)\end{array}$ & $\begin{array}{c}0.119 \\
(0.123)\end{array}$ \\
\hline 10.30 & $\begin{array}{c}0.166 \\
(0.160)\end{array}$ & $\begin{array}{c}0.183 \\
(0.183)\end{array}$ & $\begin{array}{c}0.213 \\
(0.210)\end{array}$ & $\begin{array}{c}0.275 \\
(0.262)\end{array}$ & $\begin{array}{c}0.296 \\
(0.303)\end{array}$ & $\begin{array}{c}0.370 \\
(0.382)\end{array}$ & $\begin{array}{c}0.347 \\
(0.358)\end{array}$ & $\begin{array}{c}0.325 \\
(0.317)\end{array}$ & $\begin{array}{c}0.245 \\
(0.256)\end{array}$ & $\begin{array}{c}0.169 \\
(0.169)\end{array}$ & $\begin{array}{c}0.145 \\
(0.137)\end{array}$ & $\begin{array}{c}0.144 \\
(0.144)\end{array}$ \\
\hline 11.30 & $\begin{array}{c}0.179 \\
(0.174)\end{array}$ & $\begin{array}{c}0.196 \\
(0.191)\end{array}$ & $\begin{array}{c}0.226 \\
(0.222)\end{array}$ & $\begin{array}{c}0.292 \\
(0.274)\end{array}$ & $\begin{array}{c}0.313 \\
(0.315)\end{array}$ & $\begin{array}{c}0.395 \\
(0.399)\end{array}$ & $\begin{array}{c}0.372 \\
(0.372)\end{array}$ & $\begin{array}{c}0.351 \\
(0.334)\end{array}$ & $\begin{array}{c}0.263 \\
(0.269)\end{array}$ & $\begin{array}{c}0.180 \\
(0.179)\end{array}$ & $\begin{array}{c}0.154 \\
(0.145)\end{array}$ & $\begin{array}{c}0.155 \\
(0.155)\end{array}$ \\
\hline 12.30 & $\begin{array}{c}0.179 \\
(0.172)\end{array}$ & $\begin{array}{c}0.196 \\
(0.190)\end{array}$ & $\begin{array}{c}0.226 \\
(0.226)\end{array}$ & $\begin{array}{c}0.292 \\
(0.279)\end{array}$ & $\begin{array}{c}0.313 \\
(0.318)\end{array}$ & $\begin{array}{c}0.395 \\
(0.399)\end{array}$ & $\begin{array}{c}0.372 \\
(0.365)\end{array}$ & $\begin{array}{c}0.351 \\
(0.331)\end{array}$ & $\begin{array}{c}0.263 \\
(0.263)\end{array}$ & $\begin{array}{c}0.180 \\
(0.177)\end{array}$ & $\begin{array}{c}0.154 \\
(0.146)\end{array}$ & $\begin{array}{c}0.155 \\
(0.156)\end{array}$ \\
\hline 13.30 & $\begin{array}{c}0.166 \\
(0.162)\end{array}$ & $\begin{array}{c}0.183 \\
(0.180)\end{array}$ & $\begin{array}{c}0.213 \\
(0.215)\end{array}$ & $\begin{array}{c}0.275 \\
(0.274)\end{array}$ & $\begin{array}{c}0.296 \\
(0.308)\end{array}$ & $\begin{array}{c}0.370 \\
(0.377)\end{array}$ & $\begin{array}{c}0.347 \\
(0.333)\end{array}$ & $\begin{array}{c}0.325 \\
(0.302)\end{array}$ & $\begin{array}{c}0.245 \\
(0.248)\end{array}$ & $\begin{array}{c}0.169 \\
(0.169)\end{array}$ & $\begin{array}{c}0.145 \\
(0.142)\end{array}$ & $\begin{array}{c}0.144 \\
(0.147)\end{array}$ \\
\hline 14.30 & $\begin{array}{c}0.138 \\
(0.137)\end{array}$ & $\begin{array}{c}0.156 \\
(0.160)\end{array}$ & $\begin{array}{c}0.185 \\
(0.197)\end{array}$ & $\begin{array}{c}0.241 \\
(0.250)\end{array}$ & $\begin{array}{c}0.261 \\
(0.286)\end{array}$ & $\begin{array}{c}0.322 \\
(0.340)\end{array}$ & $\begin{array}{c}0.299 \\
(0.291)\end{array}$ & $\begin{array}{c}0.277 \\
(0.265)\end{array}$ & $\begin{array}{c}0.210 \\
(0.217)\end{array}$ & $\begin{array}{c}0.147 \\
(0.154)\end{array}$ & $\begin{array}{c}0.125 \\
(0.124)\end{array}$ & $\begin{array}{c}0.119 \\
(0.125)\end{array}$ \\
\hline 15.30 & $\begin{array}{c}0.095 \\
(0.100)\end{array}$ & $\begin{array}{c}0.114 \\
(0.125)\end{array}$ & $\begin{array}{c}0.143 \\
(0.163)\end{array}$ & $\begin{array}{c}0.190 \\
(0.212)\end{array}$ & $\begin{array}{c}0.211 \\
(0.247)\end{array}$ & $\begin{array}{c}0.256 \\
(0.279)\end{array}$ & $\begin{array}{c}0.233 \\
(0.241)\end{array}$ & $\begin{array}{c}0.211 \\
(0.210)\end{array}$ & $\begin{array}{c}0.159 \\
(0.167)\end{array}$ & $\begin{array}{c}0.110 \\
(0.126)\end{array}$ & $\begin{array}{c}0.090 \\
(0.099)\end{array}$ & $\begin{array}{c}0.081 \\
(0.092)\end{array}$ \\
\hline 16.30 & $\begin{array}{c}0.040 \\
(0.051)\end{array}$ & $\begin{array}{c}0.060 \\
(0.075)\end{array}$ & $\begin{array}{c}0.087 \\
(0.113)\end{array}$ & $\begin{array}{c}0.126 \\
(0.156)\end{array}$ & $\begin{array}{c}0.148 \\
(0.189)\end{array}$ & $\begin{array}{c}0.178 \\
(0.203)\end{array}$ & $\begin{array}{c}0.158 \\
(0.174)\end{array}$ & $\begin{array}{c}0.137 \\
(0.149)\end{array}$ & $\begin{array}{c}0.098 \\
(0.118)\end{array}$ & $\begin{array}{c}0.061 \\
(0.083)\end{array}$ & $\begin{array}{c}0.042 \\
(0.055)\end{array}$ & $\begin{array}{c}0.031 \\
(0.043)\end{array}$ \\
\hline 17.30 & $\begin{array}{c}0.000 \\
(0.005)\end{array}$ & $\begin{array}{c}0.001 \\
(0.000)\end{array}$ & $\begin{array}{c}0.025 \\
(0.041)\end{array}$ & $\begin{array}{c}0.056 \\
(0.079)\end{array}$ & $\begin{array}{c}0.081 \\
(0.107)\end{array}$ & $\begin{array}{c}0.097 \\
(0.114)\end{array}$ & $\begin{array}{c}0.083 \\
(0.101)\end{array}$ & $\begin{array}{c}0.063 \\
(0.075)\end{array}$ & $\begin{array}{c}0.034 \\
(0.048)\end{array}$ & $\begin{array}{c}0.007 \\
(0.021)\end{array}$ & $\begin{array}{c}0.000 \\
(0.006)\end{array}$ & $\begin{array}{c}0.000 \\
(0.002)\end{array}$ \\
\hline Total & $\begin{array}{c}1.237 \\
(1.240)\end{array}$ & $\begin{array}{c}1.418 \\
(1.474)\end{array}$ & $\begin{array}{c}1.757 \\
(1.861)\end{array}$ & $\begin{array}{c}2.358 \\
(2.473)\end{array}$ & $\begin{array}{c}2.637 \\
(2.922)\end{array}$ & $\begin{array}{c}3.259 \\
(3.540)\end{array}$ & $\begin{array}{c}3.002 \\
(3.135)\end{array}$ & $\begin{array}{c}2.730 \\
(2.727)\end{array}$ & $\begin{array}{c}2.020 \\
(2.152)\end{array}$ & $\begin{array}{c}1.348 \\
(1.465)\end{array}$ & $\begin{array}{c}1.109 \\
(1.141)\end{array}$ & $\begin{array}{c}1.059 \\
(1.117)\end{array}$ \\
\hline
\end{tabular}

( ${ }^{*}$ measured values are in bracket).

Table 6. Estimated hourly diffuse solar radiation $\left(\mathrm{kwh} / \mathrm{m}^{2}\right)$ for Shimla.

\begin{tabular}{|c|c|c|c|c|c|c|c|c|c|c|c|c|}
\hline LAT & Jan. & Feb. & Mar. & Apr. & May & Jun. & Jul. & Aug. & Sep. & Oct. & Nov. & Dec. \\
\hline 5.30 & 0.000 & 0.000 & 0.000 & 0.000 & 0.012 & 0.023 & 0.014 & 0.002 & 0.000 & 0.000 & 0.000 & 0.000 \\
\hline 6.30 & 0.000 & 0.000 & 0.013 & 0.040 & 0.057 & 0.079 & 0.051 & 0.039 & 0.022 & 0.003 & 0.000 & 0.000 \\
\hline 7.30 & 0.022 & 0.033 & 0.049 & 0.090 & 0.108 & 0.141 & 0.090 & 0.079 & 0.063 & 0.042 & 0.022 & 0.013 \\
\hline 8.30 & 0.060 & 0.069 & 0.086 & 0.139 & 0.157 & 0.203 & 0.127 & 0.116 & 0.105 & 0.080 & 0.053 & 0.041 \\
\hline 9.30 & 0.094 & 0.101 & 0.117 & 0.181 & 0.199 & 0.256 & 0.159 & 0.149 & 0.142 & 0.112 & 0.080 & 0.065 \\
\hline 10.30 & 0.118 & 0.124 & 0.139 & 0.211 & 0.229 & 0.296 & 0.181 & 0.172 & 0.168 & 0.134 & 0.099 & 0.083 \\
\hline 11.30 & 0.131 & 0.136 & 0.150 & 0.226 & 0.244 & 0.316 & 0.193 & 0.184 & 0.182 & 0.145 & 0.108 & 0.093 \\
\hline 12.30 & 0.131 & 0.136 & 0.150 & 0.226 & 0.244 & 0.316 & 0.193 & 0.184 & 0.182 & 0.145 & 0.108 & 0.093 \\
\hline 13.30 & 0.118 & 0.124 & 0.139 & 0.211 & 0.229 & 0.296 & 0.181 & 0.172 & 0.168 & 0.134 & 0.099 & 0.083 \\
\hline 14.30 & 0.094 & 0.101 & 0.117 & 0.181 & 0.199 & 0.256 & 0.159 & 0.149 & 0.142 & 0.112 & 0.080 & 0.065 \\
\hline 15.30 & 0.060 & 0.069 & 0.086 & 0.139 & 0.157 & 0.203 & 0.127 & 0.116 & 0.105 & 0.080 & 0.053 & 0.041 \\
\hline 16.30 & 0.022 & 0.033 & 0.049 & 0.090 & 0.108 & 0.141 & 0.090 & 0.079 & 0.063 & 0.042 & 0.022 & 0.013 \\
\hline 17.30 & 0.000 & 0.000 & 0.013 & 0.040 & 0.057 & 0.079 & 0.051 & 0.039 & 0.022 & 0.003 & 0.000 & 0.000 \\
\hline Total & 0.850 & 0.927 & 1.109 & 1.772 & 2.002 & 2.605 & 1.618 & 1.481 & 1.363 & 1.031 & 0.724 & 0.591 \\
\hline
\end{tabular}


For North facing surfaces in the month having -ve declination in place of Equation (19) following relation is used:

$$
\cos \theta=\sin \delta \sin (\beta-\phi)+\cos \delta \cos \omega \cos (\beta-\phi)
$$

For North facing surfaces in the month having +ve declination

$$
\cos \theta=\sin \delta \sin (\phi-\beta)+\cos \delta \cos \omega \cos (\phi-\beta)
$$
Sun,

The angle of incidence $\theta z$ is the zenith angle of the

$$
\cos \theta z=\sin \phi \sin \delta+\cos \phi \cos \delta \cos \omega
$$

$r_{d}$ is the ratio of the diffuse radiation flux falling on the tilted surface to that falling on horizontal surface, assuming that the sky is an isotropic source of diffuse radiation,

$$
r_{d}=(1+\cos \beta) / 2
$$

Assuming that the reflection of the beam on the ground is isotropic and the reflectivity is $\rho$ the tilt factor for reflected radiation is

$$
r_{r}=\rho(1-\cos \beta) / 2
$$

The hourly tilt factor for Delhi on south facing surface has been estimated and presented in Table 7 which is in close agreement with the values given by Mani [7]. The solar radiation values for different orientations for the typical summer month May and winter month December have been calculated and are given in Tables 8 and 9. The estimated values are close to the measured values. However, Tables 8 and 9 reveal that for west orientation the estimated values in the morning are negative whereas for east orientation the evening values are negative, these values are taken to be zero.

Using this method total hourly solar radiation on south, east, west and north tilted surface for Shimla are presented in Tables 10-13 and for inclined surfaces at different tilt angles are presented in Tables 14-15. Here we made a study of ground-reflected component of radiation on inclined surfaces with varying inclination angles for Lucknow (Latitude 26.750, Longitude 80.850), Mumbai (Latitude $19.120 \mathrm{~N}$, Longitude $72.850 \mathrm{E}$ ), Calcutta (Latitude $22.650 \mathrm{~N}$, Longitude $88.350 \mathrm{E}$ ), and Pune (Latitude $18.530 \mathrm{~N}$, Longitude 73.910 E) cities of India using isotropic and anisotropic models [11]. The total solar radiation incident on a tilted surface facing south Ht with different tilt angles is then calculated using both Liu and Jordan isotropic model and Klucher's anisotropic model [12].

Table 7. Computed* and estimated hourly global radiation tilt $($ tilt = lat) factors for south facing surfaces (Azimuth zero) for Delhi.

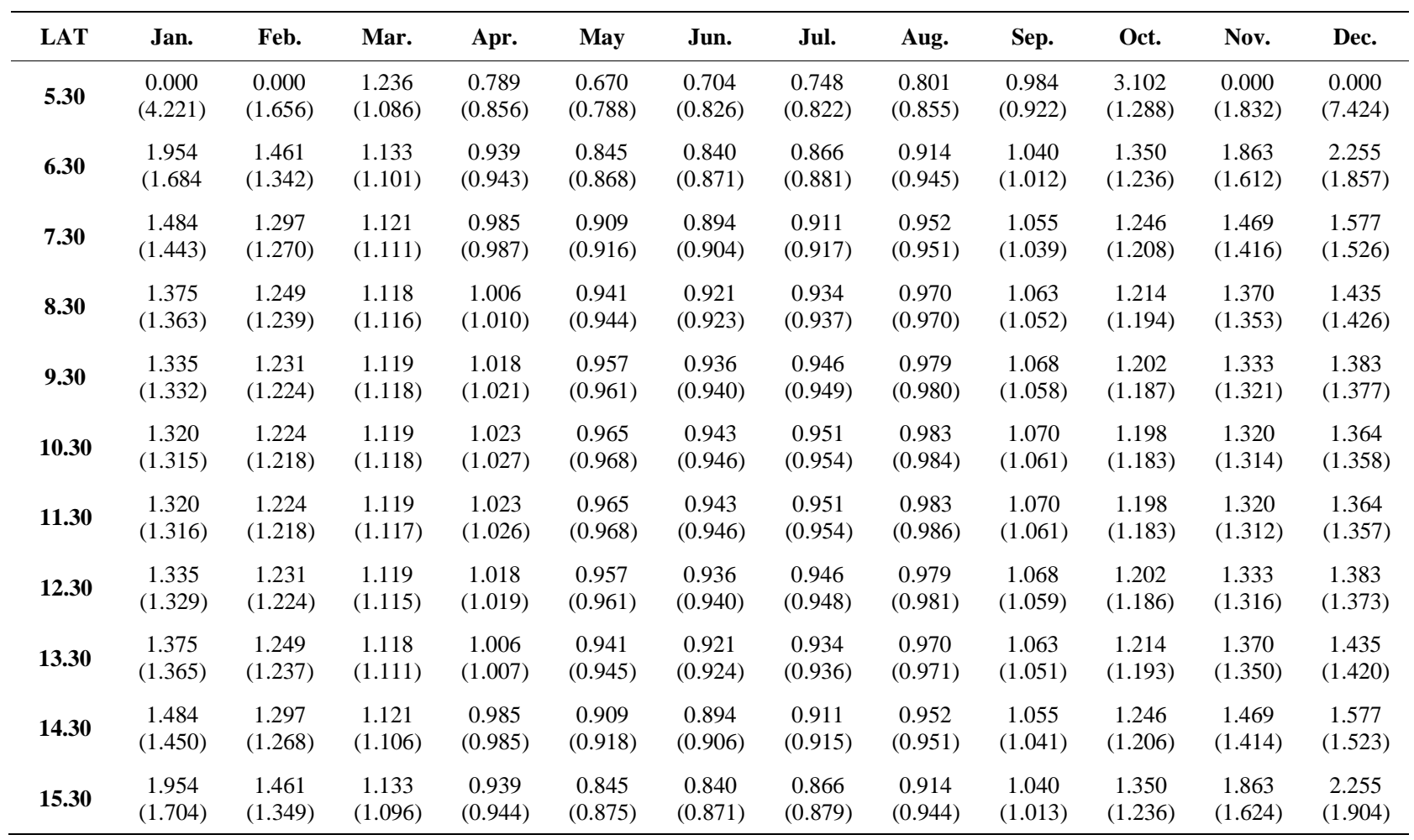

*values in brackets from Table-278, A. Mani (1980). 
Table 8. Total Estimated and measured* hourly total solar radiation $\left(\mathrm{kwh} / \mathrm{m}^{2}\right)$ on different orientation for the month for May in Delhi.

\begin{tabular}{ccccc}
\hline Is & Iw & Ie & In & LAT \\
\hline $0.000(0.017)$ & $0.000(0.017)$ & $0.484(0.017)$ & $0.000(0.017)$ & $\mathbf{5 . 3 0}$ \\
$0.000(0.075)$ & $0.000(0.075)$ & $0.493(0.300)$ & $0.000(0.128)$ & $\mathbf{6 . 3 0}$ \\
$0.057(0.133)$ & $0.000(0.133)$ & $0.559(0.491)$ & $0.057(0.178)$ & $\mathbf{7 . 3 0}$ \\
$0.160(0.180)$ & $0.000(0.180)$ & $0.587(0.563)$ & $0.160(0.184)$ & $\mathbf{8 . 3 0}$ \\
$0.253(0.258)$ & $0.000(0.214)$ & $0.559(0.545)$ & $0.253(0.214)$ & $\mathbf{9 . 3 0}$ \\
$0.325(0.321)$ & $0.003(0.238)$ & $0.472(0.459)$ & $0.325(0.238)$ & $\mathbf{1 0 . 3 0}$ \\
$0.364(0.354)$ & $0.171(0.250)$ & $0.335(0.327)$ & $0.364(0.250)$ & $\mathbf{1 1 . 3 0}$ \\
$0.364(0.353)$ & $0.335(0.327)$ & $0.171(0.251)$ & $0.364(0.251)$ & $\mathbf{1 2 . 3 0}$ \\
$0.325(0.317)$ & $0.472(0.449)$ & $0.003(0.238)$ & $0.325(0.238)$ & $\mathbf{1 3 . 3 0}$ \\
$0.253(0.256)$ & $0.559(0.522)$ & $0.000(0.215)$ & $0.253(0.215)$ & $\mathbf{1 4 . 3 0}$ \\
$0.160(0.178)$ & $0.587(0.519)$ & $0.000(0.178)$ & $0.160(0.181)$ & $\mathbf{1 5 . 3 0}$ \\
$0.057(0.130)$ & $0.559(0.432)$ & $0.000(0.130)$ & $0.057(0.168)$ & $\mathbf{1 6 . 3 0}$ \\
$0.000(0.069)$ & $0.493(0.252)$ & $0.000(0.069)$ & $0.000(0.113)$ & $\mathbf{1 7 . 3 0}$ \\
$0.000(0.014)$ & $0.386(0.117)$ & $0.000(0.014)$ & $0.000(0.051)$ & $\mathbf{1 8 . 3 0}$ \\
\hline
\end{tabular}

*Vlues are in brackets.

Table 9. Total Estimated and measured* hourly total solar radiation $\left(\mathrm{kwh} / \mathrm{m}^{2}\right)$ on different orientation for the Month of December for Delhi.

\begin{tabular}{ccccc}
\hline Is & Ie & Iw & LAT & In \\
\hline $0.000(0.001)$ & $0.000(0.002)$ & $0.000(0.001)$ & $\mathbf{5 . 3 0}$ & $0.000(0.001)$ \\
$0.296(0.199)$ & $0.470(0.310)$ & $0.000(0.031)$ & $\mathbf{6 . 3 0}$ & $0.000(0.031)$ \\
$0.440(0.407)$ & $0.507(0.472)$ & $0.000(0.072)$ & $\mathbf{7 . 3 0}$ & $0.000(0.072)$ \\
$0.578(0.565)$ & $0.484(0.477)$ & $0.000(0.104)$ & $\mathbf{8 . 3 0}$ & $0.025(0.104)$ \\
$0.688(0.662)$ & $0.388(0.378)$ & $0.000(0.126)$ & $\mathbf{9 . 3 0}$ & $0.132(0.126)$ \\
$0.749(0.710)$ & $0.233(0.226)$ & $0.047(0.138)$ & $\mathbf{1 0 . 3 0}$ & $0.193(0.138)$ \\
$0.749(0.709)$ & $0.047(0.139)$ & $0.233(0.226)$ & $\mathbf{1 1 . 3 0}$ & $0.211(0.139)$ \\
$0.688(0.652)$ & $0.000(0.127)$ & $0.388(0.374)$ & $\mathbf{1 2 . 3 0}$ & $0.189(0.127)$ \\
$0.578(0.552)$ & $0.000(0.104)$ & $0.484(0.466)$ & $\mathbf{1 3 . 3 0}$ & $0.136(0.104)$ \\
$0.440(0.399)$ & $0.000(0.072)$ & $0.507(0.462)$ & $\mathbf{1 4 . 3 0}$ & $0.069(0.072)$ \\
$0.296(0.211)$ & $0.000(0.030)$ & $0.470(0.330)$ & $\mathbf{1 5 . 3 0}$ & $0.009(0.030)$ \\
$0.000(0.002)$ & $0.000(0.001)$ & $0.000(0.001)$ & $\mathbf{1 6 . 3 0}$ & $0.000(0.001)$ \\
\hline
\end{tabular}

*Values are in brackets 
Table 10. Estimated total hourly solar radiation $\left(\mathrm{kwh} / \mathrm{m}^{2}\right)$ on south orientation for Shimla.

\begin{tabular}{|c|c|c|c|c|c|c|c|c|c|c|c|c|}
\hline Hour angle & Jan. & Feb. & Mar. & Apr. & May & Jun. & Jul. & Aug. & Sep. & Oct. & Nov. & Dec. \\
\hline 97.50 & 0.000 & 0.000 & 0.000 & 0.000 & 0.000 & 0.000 & 0.000 & 0.000 & 0.000 & 0.000 & 0.000 & 0.000 \\
\hline 67.50 & 0.153 & 0.128 & 0.097 & 0.078 & 0.041 & 0.051 & 0.036 & 0.042 & 0.080 & 0.162 & 0.193 & 0.164 \\
\hline 52.50 & 0.244 & 0.207 & 0.166 & 0.156 & 0.104 & 0.112 & 0.065 & 0.072 & 0.143 & 0.264 & 0.304 & 0.260 \\
\hline 37.50 & 0.334 & 0.282 & 0.230 & 0.229 & 0.163 & 0.168 & 0.088 & 0.096 & 0.200 & 0.362 & 0.412 & 0.355 \\
\hline 22.50 & 0.406 & 0.341 & 0.280 & 0.286 & 0.208 & 0.210 & 0.105 & 0.114 & 0.245 & 0.439 & 0.498 & 0.431 \\
\hline 7.50 & 0.445 & 0.373 & 0.307 & 0.317 & 0.233 & 0.234 & 0.113 & 0.123 & 0.269 & 0.482 & 0.546 & 0.473 \\
\hline-7.50 & 0.445 & 0.373 & 0.307 & 0.317 & 0.233 & 0.234 & 0.113 & 0.123 & 0.269 & 0.482 & 0.546 & 0.473 \\
\hline-22.50 & 0.406 & 0.341 & 0.280 & 0.286 & 0.208 & 0.210 & 0.105 & 0.114 & 0.245 & 0.439 & 0.498 & 0.431 \\
\hline-37.50 & 0.334 & 0.282 & 0.230 & 0.229 & 0.163 & 0.168 & 0.088 & 0.096 & 0.200 & 0.362 & 0.412 & 0.355 \\
\hline-52.50 & 0.244 & 0.207 & 0.166 & 0.156 & 0.104 & 0.112 & 0.065 & 0.072 & 0.143 & 0.264 & 0.304 & 0.260 \\
\hline-67.50 & 0.153 & 0.128 & 0.097 & 0.078 & 0.041 & 0.051 & 0.036 & 0.042 & 0.080 & 0.162 & 0.193 & 0.164 \\
\hline-82.5 & 0.000 & 0.000 & 0.033 & 0.006 & -.018 & 0.000 & 0.005 & 0.009 & 0.022 & 0.069 & 0.000 & 0.000 \\
\hline-97.5 & 0.000 & 0.000 & 0.000 & 0.000 & 0.000 & 0.000 & 0.000 & 0.000 & 0.000 & 0.000 & 0.000 & 0.000 \\
\hline Total & 3.167 & 2.662 & 2.226 & 2.148 & 1.481 & 1.551 & 0.823 & 0.912 & 1.918 & 3.554 & 3.907 & 3.364 \\
\hline
\end{tabular}

Table 11. Estimated total hourly solar radiation $\left(\mathrm{kwh} / \mathrm{m}^{2}\right)$ on east orientation for Shimla.

\begin{tabular}{|c|c|c|c|c|c|c|c|c|c|c|c|c|}
\hline Hour angle & Jan. & Feb. & Mar. & Apr. & May & Jun. & Jul. & Aug. & Sep. & Oct. & Nov. & Dec. \\
\hline 97.50 & 0.000 & 0.000 & 0.000 & 0.000 & 0.213 & 0.171 & 0.098 & 0.099 & 0.000 & 0.000 & 0.000 & 0.000 \\
\hline 82.50 & 0.000 & 0.000 & 0.242 & 0.295 & 0.269 & 0.227 & 0.120 & 0.119 & 0.220 & 0.308 & 0.000 & 0.000 \\
\hline 67.50 & 0.259 & 0.268 & 0.283 & 0.348 & 0.311 & 0.273 & 0.134 & 0.134 & 0.258 & 0.376 & 0.115 & 0.261 \\
\hline 37.50 & 0.288 & 0.281 & 0.284 & 0.359 & 0.319 & 0.305 & 0.138 & 0.137 & 0.265 & 0.386 & 0.246 & 0.292 \\
\hline 22.50 & 0.240 & 0.232 & 0.234 & 0.306 & 0.274 & 0.280 & 0.127 & 0.125 & 0.225 & 0.313 & 0.222 & 0.240 \\
\hline 7.50 & 0.152 & 0.151 & 0.157 & 0.218 & 0.202 & 0.229 & 0.108 & 0.104 & 0.161 & 0.196 & 0.153 & 0.149 \\
\hline-7.50 & 0.044 & 0.051 & 0.063 & 0.109 & 0.112 & 0.160 & 0.083 & 0.078 & 0.081 & 0.055 & 0.061 & 0.035 \\
\hline-22.50 & 0.000 & 0.000 & 0.000 & 0.000 & 0.018 & 0.083 & 0.054 & 0.047 & 0.000 & 0.000 & 0.000 & 0.000 \\
\hline-37.50 & 0.000 & 0.000 & 0.000 & 0.000 & 0.000 & 0.008 & 0.023 & 0.014 & 0.000 & 0.000 & 0.000 & 0.000 \\
\hline-52.50 & 0.000 & 0.000 & 0.000 & 0.000 & 0.000 & 0.000 & 0.000 & 0.000 & 0.000 & 0.000 & 0.000 & 0.000 \\
\hline-67.50 & 0.000 & 0.000 & 0.000 & 0.000 & 0.000 & 0.000 & 0.000 & 0.000 & 0.000 & 0.000 & 0.000 & 0.000 \\
\hline-82.5 & 0.000 & 0.000 & 0.000 & 0.000 & 0.000 & 0.000 & 0.000 & 0.000 & 0.000 & 0.000 & 0.000 & 0.000 \\
\hline-97.5 & 0.000 & 0.000 & 0.000 & 0.000 & 0.000 & 0.000 & 0.000 & 0.000 & 0.000 & 0.000 & 0.000 & 0.000 \\
\hline Total & 1.276 & 1.275 & 1.561 & 2.007 & 2.047 & 2.038 & 1.026 & 0.995 & 1.484 & 2.040 & 1.008 & 1.275 \\
\hline
\end{tabular}


Table 12. Estimated total hourly solar radiation $\left(\mathrm{kwh} / \mathrm{m}^{2}\right)$ on west orientation for Shimla.

\begin{tabular}{|c|c|c|c|c|c|c|c|c|c|c|c|c|}
\hline Hour angle & Jan. & Feb. & Mar. & Apr. & May & Jun. & Jul. & Aug. & Sep. & Oct. & Nov. & Dec. \\
\hline 97.50 & 0.000 & 0.000 & 0.000 & 0.000 & 0.000 & 0.000 & 0.000 & 0.000 & 0.000 & 0.000 & 0.000 & 0.000 \\
\hline 82.50 & 0.000 & 0.000 & 0.000 & 0.000 & 0.000 & 0.000 & 0.000 & 0.000 & 0.000 & 0.000 & 0.000 & 0.000 \\
\hline 67.50 & 0.000 & 0.000 & 0.000 & 0.000 & 0.000 & 0.000 & 0.000 & 0.000 & 0.000 & 0.000 & 0.000 & 0.000 \\
\hline 37.50 & 0.000 & 0.000 & 0.000 & 0.000 & 0.000 & 0.008 & 0.023 & 0.014 & 0.000 & 0.000 & 0.000 & 0.000 \\
\hline 22.50 & 0.000 & 0.000 & 0.000 & 0.000 & 0.018 & 0.083 & 0.054 & 0.047 & 0.000 & 0.000 & 0.000 & 0.000 \\
\hline 7.50 & 0.044 & 0.051 & 0.063 & 0.109 & 0.112 & 0.160 & 0.083 & 0.078 & 0.081 & 0.055 & 0.036 & 0.035 \\
\hline-7.50 & 0.152 & 0.151 & 0.157 & 0.218 & 0.202 & 0.229 & 0.108 & 0.104 & 0.161 & 0.196 & 0.178 & 0.149 \\
\hline-22.50 & 0.240 & 0.232 & 0.234 & 0.306 & 0.274 & 0.280 & 0.127 & 0.125 & 0.225 & 0.313 & 0.295 & 0.240 \\
\hline-37.50 & 0.288 & 0.281 & 0.284 & 0.359 & 0.319 & 0.305 & 0.138 & 0.137 & 0.265 & 0.386 & 0.366 & 0.292 \\
\hline-52.50 & 0.293 & 0.292 & 0.299 & 0.373 & 0.330 & 0.302 & 0.141 & 0.140 & 0.275 & 0.406 & 0.381 & 0.297 \\
\hline-67.50 & 0.259 & 0.268 & 0.283 & 0.348 & 0.311 & 0.273 & 0.134 & 0.134 & 0.258 & 0.376 & 0.345 & 0.261 \\
\hline-82.5 & 0.000 & 0.000 & 0.242 & 0.295 & 0.269 & 0.227 & 0.120 & 0.119 & 0.220 & 0.308 & 0.000 & 0.000 \\
\hline-97.5 & 0.000 & 0.000 & 0.000 & 0.000 & 0.000 & 0.000 & 0.000 & 0.000 & 0.000 & 0.000 & 0.000 & 0.000 \\
\hline Total & 1.276 & 1.275 & 1.561 & 2.007 & 1.834 & 1.867 & 0.929 & 0.896 & 1.484 & 2.040 & 1.601 & 1.275 \\
\hline
\end{tabular}

Table 13. Estimated total hourly solar radiation $\left(\mathrm{kwh} / \mathrm{m}^{2}\right)$ on north orientation for Shimla.

\begin{tabular}{|c|c|c|c|c|c|c|c|c|c|c|c|c|}
\hline Hour angle & Jan. & Feb. & Mar. & Apr. & May & Jun. & Jul. & Aug. & Sep. & Oct. & Nov. & Dec. \\
\hline 97.50 & 0.000 & 0.000 & 0.000 & 0.000 & 0.000 & 0.000 & 0.000 & 0.000 & 0.000 & 0.000 & 0.000 & 0.000 \\
\hline 82.50 & 0.000 & 0.000 & 0.017 & 0.006 & 0.000 & 0.000 & 0.005 & 0.009 & 0.022 & 0.000 & 0.000 & 0.000 \\
\hline 67.50 & 0.000 & 0.022 & 0.078 & 0.078 & 0.041 & 0.051 & 0.036 & 0.042 & 0.080 & 0.051 & 0.000 & 0.000 \\
\hline 37.50 & 0.098 & 0.145 & 0.206 & 0.229 & 0.163 & 0.168 & 0.088 & 0.096 & 0.200 & 0.219 & 0.131 & 0.080 \\
\hline 22.50 & 0.148 & 0.193 & 0.255 & 0.286 & 0.208 & 0.210 & 0.105 & 0.114 & 0.245 & 0.285 & 0.191 & 0.128 \\
\hline 7.50 & 0.175 & 0.220 & 0.281 & 0.317 & 0.233 & 0.234 & 0.113 & 0.123 & 0.269 & 0.321 & 0.225 & 0.156 \\
\hline-7.50 & 0.175 & 0.220 & 0.281 & 0.317 & 0.233 & 0.234 & 0.113 & 0.123 & 0.269 & 0.321 & 0.225 & 0.156 \\
\hline-22.50 & 0.148 & 0.193 & 0.255 & 0.286 & 0.208 & 0.210 & 0.105 & 0.114 & 0.245 & 0.285 & 0.191 & 0.128 \\
\hline-37.50 & 0.098 & 0.145 & 0.206 & 0.229 & 0.163 & 0.168 & 0.088 & 0.096 & 0.200 & 0.219 & 0.131 & 0.080 \\
\hline-52.50 & 0.039 & 0.084 & 0.144 & 0.156 & 0.104 & 0.112 & 0.065 & 0.072 & 0.143 & 0.136 & 0.057 & 0.022 \\
\hline-67.50 & 0.000 & 0.022 & 0.078 & 0.078 & 0.041 & 0.051 & 0.036 & 0.042 & 0.080 & 0.051 & 0.000 & 0.000 \\
\hline-82.5 & 0.000 & 0.000 & 0.017 & 0.006 & 0.000 & 0.000 & 0.005 & 0.009 & 0.022 & 0.000 & 0.000 & 0.000 \\
\hline-97.5 & 0.000 & 0.000 & 0.000 & 0.000 & 0.000 & 0.000 & 0.000 & 0.000 & 0.000 & 0.000 & 0.000 & 0.000 \\
\hline Total & 0.921 & 1.329 & 1.962 & 2.148 & 1.499 & 1.551 & 0.823 & 0.912 & 1.918 & 2.025 & 1.206 & 0.771 \\
\hline
\end{tabular}


Table 14. Estimated total hourly solar radiation $\left(\mathrm{kwh} / \mathrm{m}^{2}\right)$ on south orientation $(\mathrm{tilt}=$ lat $)$ for Shimla.

\begin{tabular}{|c|c|c|c|c|c|c|c|c|c|c|c|c|}
\hline Hour angle & Jan. & Feb. & Mar. & Apr. & May & Jun. & Jul. & Aug. & Sep. & Oct. & Nov. & Dec. \\
\hline 97.50 & 0.000 & 0.000 & 0.000 & 0.000 & 0.000 & 0.000 & 0.000 & 0.000 & 0.000 & 0.000 & 0.000 & 0.000 \\
\hline 82.50 & 0.000 & 0.000 & 0.042 & 0.070 & 0.076 & 0.000 & 0.053 & 0.044 & 0.045 & 0.043 & 0.000 & 0.000 \\
\hline 67.50 & 0.120 & 0.130 & 0.145 & 0.197 & 0.187 & 0.051 & 0.105 & 0.098 & 0.143 & 0.181 & 0.158 & 0.118 \\
\hline 52.50 & 0.244 & 0.246 & 0.256 & 0.334 & 0.303 & 0.112 & 0.152 & 0.147 & 0.246 & 0.337 & 0.311 & 0.244 \\
\hline 37.50 & 0.369 & 0.359 & 0.361 & 0.461 & 0.410 & 0.168 & 0.190 & 0.187 & 0.342 & 0.488 & 0.463 & 0.373 \\
\hline 22.50 & 0.471 & 0.449 & 0.442 & 0.560 & 0.492 & 0.210 & 0.217 & 0.215 & 0.416 & 0.607 & 0.586 & 0.477 \\
\hline 7.50 & 0.527 & 0.498 & 0.487 & 0.614 & 0.537 & 0.234 & 0.232 & 0.230 & 0.456 & 0.673 & 0.655 & 0.536 \\
\hline-7.50 & 0.527 & 0.498 & 0.487 & 0.614 & 0.537 & 0.234 & 0.232 & 0.230 & 0.456 & 0.673 & 0.655 & 0.536 \\
\hline-22.50 & 0.471 & 0.449 & 0.442 & 0.560 & 0.492 & 0.210 & 0.217 & 0.215 & 0.416 & 0.607 & 0.586 & 0.477 \\
\hline-37.50 & 0.369 & 0.359 & 0.361 & 0.461 & 0.410 & 0.168 & 0.190 & 0.187 & 0.342 & 0.488 & 0.463 & 0.373 \\
\hline-52.50 & 0.244 & 0.246 & 0.256 & 0.334 & 0.303 & 0.112 & 0.152 & 0.147 & 0.246 & 0.337 & 0.311 & 0.244 \\
\hline-67.50 & 0.120 & 0.130 & 0.145 & 0.197 & 0.187 & 0.051 & 0.105 & 0.098 & 0.143 & 0.181 & 0.158 & 0.118 \\
\hline-82.5 & 0.000 & 0.000 & 0.042 & 0.070 & 0.076 & 0.000 & 0.053 & 0.044 & 0.045 & 0.043 & 0.000 & 0.000 \\
\hline-97.5 & 0.000 & 0.000 & 0.000 & 0.000 & 0.000 & 0.000 & 0.000 & 0.000 & 0.000 & 0.000 & 0.000 & 0.000 \\
\hline Total & 3.460 & 3.362 & 3.468 & 4.472 & 4.010 & 1.551 & 1.897 & 1.845 & 3.296 & 4.658 & 4.347 & 3.498 \\
\hline
\end{tabular}

Table 15. Estimated total hourly solar radiation $\left(\mathrm{kwh} / \mathrm{m}^{2}\right)$ on south orientation $(\mathrm{tilt}=\mathrm{lat}+15)$ for Shimla.

\begin{tabular}{|c|c|c|c|c|c|c|c|c|c|c|c|c|}
\hline Hour angle & Jan. & Feb. & Mar. & Apr. & May & Jun. & Jul. & Aug. & Sep. & Oct. & Nov. & Dec. \\
\hline 97.50 & 0.000 & 0.000 & 0.000 & 0.000 & 0.000 & 0.000 & 0.000 & 0.000 & 0.000 & 0.000 & 0.000 & 0.000 \\
\hline 82.50 & 0.000 & 0.000 & 0.043 & 0.055 & 0.052 & 0.066 & 0.041 & 0.036 & 0.042 & 0.056 & 0.000 & 0.000 \\
\hline 67.50 & 0.142 & 0.141 & 0.143 & 0.176 & 0.156 & 0.165 & 0.090 & 0.088 & 0.135 & 0.192 & 0.184 & 0.144 \\
\hline 37.50 & 0.392 & 0.368 & 0.353 & 0.428 & 0.367 & 0.364 & 0.172 & 0.172 & 0.327 & 0.494 & 0.491 & 0.402 \\
\hline 22.50 & 0.494 & 0.456 & 0.432 & 0.523 & 0.445 & 0.437 & 0.198 & 0.199 & 0.398 & 0.611 & 0.615 & 0.508 \\
\hline 7.50 & 0.550 & 0.505 & 0.475 & 0.575 & 0.488 & 0.477 & 0.211 & 0.213 & 0.437 & 0.676 & 0.683 & 0.567 \\
\hline-7.50 & 0.550 & 0.505 & 0.475 & 0.575 & 0.488 & 0.477 & 0.211 & 0.213 & 0.437 & 0.676 & 0.683 & 0.567 \\
\hline-22.50 & 0.494 & 0.456 & 0.432 & 0.523 & 0.445 & 0.437 & 0.198 & 0.199 & 0.398 & 0.611 & 0.615 & 0.508 \\
\hline-37.50 & 0.392 & 0.368 & 0.353 & 0.428 & 0.367 & 0.364 & 0.172 & 0.172 & 0.327 & 0.494 & 0.491 & 0.402 \\
\hline-52.50 & 0.267 & 0.256 & 0.251 & 0.307 & 0.266 & 0.268 & 0.135 & 0.134 & 0.235 & 0.345 & 0.338 & 0.272 \\
\hline-67.50 & 0.142 & 0.141 & 0.143 & 0.176 & 0.156 & 0.165 & 0.090 & 0.088 & 0.135 & 0.192 & 0.184 & 0.144 \\
\hline-82.5 & 0.000 & 0.000 & 0.043 & 0.055 & 0.052 & 0.066 & 0.041 & 0.036 & 0.042 & 0.056 & 0.000 & 0.000 \\
\hline-97.5 & 0.000 & 0.000 & 0.000 & 0.000 & 0.000 & 0.000 & 0.000 & 0.000 & 0.000 & 0.000 & 0.000 & 0.000 \\
\hline Total & 3.689 & 3.452 & 3.392 & 4.130 & 3.549 & 3.552 & 1.693 & 1.685 & 3.148 & 4.749 & 4.623 & 3.785 \\
\hline
\end{tabular}




\section{Conclusions}

The mean hourly global solar radiation has been estimated using daily integration approach by Gueymard [2] for Delhi, which accurately predicts the monthly average hourly global solar radiation using model [1]. The hourly diffuse solar radiation has been calculated by improving the method of Orgill and Hollands [3], which gives good fit. The tilt factor for south surfaces has been calculated using method of Klein [4] and hourly total solar radiation on the inclined south surfaces for Delhi and Shimla has been calculated. The values of tilt factor are found to be close to the values given by Mani [7] except for early morning and late evening. The method presented can be used to calculate hourly global, diffuse solar radiation for horizontal surfaces and total solar radiation on inclined \& vertical surfaces at different orientations with greater accuracy for any location.

\section{REFERENCES}

[1] S. S. Chandel, R. K. Aggarwal and A. N. Pandey, "New Correlation to Estimate Global Solar Radiation on Horizontal Surfaces Using Sunshine Hour and Temperature Data for Indian Sites,” Solar Energy Engineering Journal, Vol. 127, No. 3, 2005, pp. 417-420. doi:10.1115/1.1877512

[2] C. Gueymard, "Prediction and Performance Assessment of Mean Hourly Global Radiation,” Solar Energy, Vol. 68, No. 3, 2000, pp. 285-303. doi:10.1016/S0038-092X(99)00070-5

[3] J. F. Orgill and K. G. Hollands T, "Correlation Equation for Hourly Diffuse Radiation on Horizontal Surfaces,"
Solar energy, Vol. 19, No. 4, 1977, pp. 357-359. doi:10.1016/0038-092X(77)90006-8

[4] S. A. Klein, "Calculation of Monthly Average Insolation on Tilted Surfaces," Solar Energy, Vol. 19, 1977, pp 325-329. doi:10.1016/0038-092X(77)90001-9

[5] A. Whillier, "The Determination of Hourly Values of Total Solar Radiation from Daily Summations," Theoretical and Applied Climatology, Vol. 7, No. 2, pp. 197-204.

[6] B. Y. H. Liu and R. C. Jordan, "The Inter Relationship and Characteristics Distribution of Direct, Diffuse and Total Solar Radiation,” Solar Energy, Vol. 4, No. 3, 1960, pp. 1-19. doi:10.1016/0038-092X(60)90062-1

[7] A. Mani, "Handbook of Solar Radiation Data for India," Allied Publishers Pvt. Ltd., New Delhi, 1980.

[8] J. A. Duffie and W. A. Beckman, "Solar Engineering of Thermal Process,” John Willy \& Sons, New York, 1980.

[9] N. Robinson, “Solar Radiation,” Elsevier, New York, 1966.

[10] K. J. A. Revfein, “A Simple Procedure for Estimating Global Daily Radiation on Any Surfaces,” Journal of Applied Meteorology, Vol. 17, No. 8, 1978, pp. 1126-1131. doi:10.1175/1520-0450(1978)017<1126:ASPFEG>2.0.C $\mathrm{O} ; 2$

[11] K. Katiyar and C. K. Panday, "Study of Ground-Reflected Component and Its Contribution in Diffuse Solar Radiation Incident on Inclined Surfaces over India,” International Journal of Energy and Environment, Vol. 1, No. 3, 2010, pp. 547-554.

[12] A. A. El-Sebaii, F. S. Al-Hazmi, A. A. Al-Ghamdi and S. J. Yaghmour, "Global, Direct and Diffuse Solar Radiation on Horizontal and Tilted Surfaces in Jeddah, Saudi Arabia,” Applied Energy, Vol. 87, No. 2, 2010, pp. 568-576. doi:10.1016/j.apenergy.2009.06.032

\section{Nomenclature}

$\mathrm{G}_{\mathrm{h}}=$ hourly solar radiation $\left(\mathrm{KWh} / \mathrm{m}^{2}\right)$

$\mathrm{G}_{0}=$ extra terrestrial solar radiation $\left(\mathrm{KWh} / \mathrm{m}^{2}\right)$

$\mathrm{Gsc}=$ solar constant $1.357 \mathrm{KW} / \mathrm{m}^{2}$

$\mathrm{c}=$ regression coefficient

$\mathrm{d}=$ regression coefficient

$\omega_{\mathrm{s}}=$ sunrise hour angle (degrees)

$\omega=$ hour angle measured from solar noon (degrees)

$\varphi=$ latitude of the place (degrees)

$\delta=$ declination angle (degrees)

$\mathrm{h}_{\mathrm{o}}=$ solar elevation outside of the atmosphere (degrees)

$\mathrm{S}_{0}=$ day length (Hrs.)

$\mathrm{P}=$ atmospheric pressure at the site $(\mathrm{K} \mathrm{Pa})$

$\mathrm{P}_{0}=$ standard atmospheric pressure $(\mathrm{K} \mathrm{Pa})$

$\mathrm{H}=$ hourly solar elevation $\left(\mathrm{KWh} / \mathrm{m}^{2}\right)$
$\mathrm{D}_{\mathrm{h}}=$ mean hourly diffuse solar radiation $\left(\mathrm{KWh} / \mathrm{m}^{2}\right)$

$\mathrm{r}=$ ground surface reflectivity

$\mathrm{b}=$ slope (degrees)

$\mathrm{r}_{\mathrm{b}}=$ tilt factor for beam radiation

$\mathrm{q}=$ angle of incidence of beam radiation (degrees)

$\mathrm{g}=$ surface azimuth angle (degrees)

$\mathrm{q}_{\mathrm{z}}=$ zenith angle of the Sun (degrees)

$r_{d}=$ tilt factor for diffuse radiation

$\mathrm{k}_{\mathrm{T}}=$ clearness index

$\mathrm{R}=$ global radiation tilt factor

$\mathrm{a}^{1}=$ regression constant

$\mathrm{a}^{2}=$ regression constant

$\mathrm{G}_{\mathrm{OH}}=$ hourly extra terrestrial solar radiation $\left(\mathrm{KWh} / \mathrm{m}^{2}\right)$

$\mathrm{G}_{\mathrm{TH}}=$ total hourly solar radiation on tilt surface $\left(\mathrm{KWh} / \mathrm{m}^{2}\right)$ 